\section{FETAL GROWTH, PRETERM BIRTH, NEONATAL STRESS AND RISK FOR CNS TUMORS IN CHILDREN: A NORDIC POPULATIONAND REGISTER-BASED CASE- CONTROL STUDY}

\section{L.S. Schmidt ${ }^{1}$, J. Schüz ${ }^{1}$, P. Lähteenmäki², C. Träger ${ }^{3}$, T. Stockland ${ }^{4}$, G. Gustafson ${ }^{3}$, L. Hjalgrim ${ }^{5}$, A. Sehested ${ }^{5}$, C. Johansen ${ }^{1}$, K. Schmiegelow ${ }^{5}$ \\ ${ }^{1}$ Institute of Cancer Epidemiology, The Danish Cancer Society, Copenhagen, Denmark, ${ }^{2}$ Department of Pediatrics, Turku University Hospital, Turku, Finland, ${ }^{3}$ Childhood Cancer Research Unit, Karolinska Institute, Karolinska University Hospital, Stockholm, Sweden, ${ }^{4}$ Department of Pediatrics, University Hospital of North Norway, Tromsø, Norway, ${ }^{5}$ Department of Pediatrics, Copenhagen University Hospital, \\ Rigshospitalet, Copenhagen, Denmark}

We investigated the association between indicators of fetal growth and neonatal stress and CNS tumours diagnosed in the Denmark, Sweden, Norway and Finland in 1985-2006, using data from national cancer registries and medical birth registries. We included 3,443 children with primary CNS tumours and 16169 individually matched controls drawn randomly from the national population registries.

We observed a U-shaped relation between risk for CNS tumors and birthweight, at $>4.5 \mathrm{~kg}$ (OR 1.27; $95 \% \mathrm{Cl}, 1.03-1.55)$ and $<2.0 \mathrm{~kg}(\mathrm{OR}, 1.50 ; 95 \%$ $\mathrm{Cl}$, 1.13-1.99), the latter being attenuated after adjustment for gestational age. Moreover, small-forgestational age (OR, 1.28; 95\% Cl, 0.98-1.66) and large-forgestationalage (OR, 1.26; $95 \% \mathrm{Cl}$, 1.02$1.55)$ were both associated with CNS tumors. The OR for preterm births was increased per 1-week decrease in gestational age (OR, 1.58; 95\% Cl, 1.04-2.44). Increased Ors were also observed for head circumference $>38 \mathrm{~cm}(1.80 ; 95 \% \mathrm{Cl}, 1.18$ 2.74), 5-minute Apgar score < $7(1.44 ; 95 \% \mathrm{Cl}$, $0.98-2.12)$, and breech presentation $(1.33 ; 95 \% \mathrm{Cl}$, 1.04-1.69). The observed associations varied little by histologic subgroup.

This study provides evidence that several intrauterine and neonatal factors are associated with higher risk of CNS tumours, indicating an early onset or, alternatively, potentially harmful exposures in the neonatal period that might be preventable.

\section{NURSE-LED TELEPHONE REFERRAL TO PAEDIATRIC EMERGENCY AND ASSESSMENT UNITS \\ D. Hausted, M. Ørnekoll, C.S. Jensen \\ Department of Paediatrics, Aarhus University Hospital, Skejby, Aarhus, Denmark}

Background: An increasing number of acute referrals have led to unsatisfactory working conditions and a degradation of the service to the families attending paediatric emergency and assessment units. The usual way of refering for the general practitioner (GP) was by telephone to the resident assistant. This study investigated if a nurse-led telephone referral system would optimize conditions for patients and staff.

Aim:

- To create optimal continuity of care

- Optimise service to Gps' and other collaborators

- Improve quality of care to the families

- To improve working conditions in the PEAU

Method: The project period was from October to December 2009. A team of paediatric nurses were in charge of the referral system with backup from the attending physician. Prior to the project the nurses were introduced to a guideline which included a model to ensure efficient and accurate reception and transmission of information - the ISBAR model. The project was evaluated using an electronic questionnaire and focus group interviews.

Findings: Nurse-led telephone referral helped to create:

- Optimal continuity of care as the nurses in charge of referral had a better overview

- Improved working procedures as information about the children were already documented prior to arrival to the PEAU

- Better working environment as staff was able to examine the children and talk to the families without interruptions

- Improved quality of the referral system accoridng to the GPs 